\title{
Is There Utility in Using Mastoid Pressure Dressing in Children Undergoing a Mastoidectomy?
}

\author{
Shadi Shinnawi ${ }^{1}$, Arie Gordin ${ }^{1}$, Iyad Zaroura ${ }^{1}$, Bahaa Rafoul ${ }^{1}$, Majd Khoury ${ }^{1}$, and \\ Mauricio Cohen-Vaizer ${ }^{1}$ \\ ${ }^{1}$ Rambam Health Care Campus
}

October 4, 2021

\begin{abstract}
Objectives: To assess the efficacy of avoiding mastoid pressure dressing (MPD) on children as a means of preventing discomfort and postoperative pain. Design: A retrospective controlled study. Setting: All operations were carried out by experienced surgeons using standard techniques, whose custom, not the gravity of any individual case, dictated the use of MPD. Participants: children who underwent mastoidectomy for inflammatory middle ear diseases at a tertiary centre from 2010-2020. Main outcome measures: Wound-related complications and visual analog scale (VAS) pain scores at discharge were compared between children who had a MPD applied following surgery and those who did not. Results: 119 cases were included. The demographic characteristics of the patients and surgical techniques employed similar for both groups. There were 91 patients in the MPD group and 28 in the non-mastoid dressing (NMPD) group. In the MPD group, 5 patients developed minor wound dehiscence, 8 experienced surgical site infections (SSI), and one patient developed a keloid. In the NMPD group, one patient had a SSI, while another had a local hematoma. Therefore, there were no differences between the groups in relation to postoperative complications $(\mathrm{p}=0.47)$. Despite these similitudes, the NMPD patients suffered less postoperative pain, as measured by the VAS ( $\mathrm{p}=.02$ ). Conclusions: This study shows that no significant benefit is derived from using a MPD after mastoidectomy in children. Surgeons should adhere to principles of appropriate haemostasis and wound closure to prevent postoperative wound complications. Our study supports the abandonment of routine MPD on children following mastoidectomy.
\end{abstract}

\section{Is There Utility in Using Mastoid Pressure Dressing in Children Undergoing a Mastoidectomy?}

\section{Abstract}

Objectives: To assess the efficacy of avoiding mastoid pressure dressing (MPD) on children as a means of preventing discomfort and postoperative pain.

Design: A retrospective controlled study.

Setting: All operations were carried out by experienced surgeons using standard techniques, whose custom, not the gravity of any individual case, dictated the use of MPD.

Participants: children who underwent mastoidectomy for inflammatory middle ear diseases at a tertiary centre from 2010-2020.

Main outcome measures: Wound-related complications and visual analog scale (VAS) pain scores at discharge were compared between children who had a MPD applied following surgery and those who did not.

Results: 119 cases were included. The demographic characteristics of the patients and surgical techniques employed similar for both groups. There were 91 patients in the MPD group and 28 in the non-mastoid dressing (NMPD) group. In the MPD group, 5 patients developed minor wound dehiscence, 8 experienced surgical site infections (SSI), and one patient developed a keloid. In the NMPD group, one patient had 
a SSI, while another had a local hematoma. Therefore, there were no differences between the groups in relation to postoperative complications $(\mathrm{p}=0.47)$. Despite these similitudes, the NMPD patients suffered less postoperative pain, as measured by the VAS $(\mathrm{p}=.02)$.

Conclusions : This study shows that no significant benefit is derived from using a MPD after mastoidectomy in children. Surgeons should adhere to principles of appropriate haemostasis and wound closure to prevent postoperative wound complications. Our study supports the abandonment of routine MPD on children following mastoidectomy.

\section{Key points}

* Following mastoid surgery, the application of MPD is believed to minimize postoperative complications, such as hematoma, seroma, and wound infection.

* MPD can cause severe discomfort, headaches, stress associated with their removal or pressure scaring, and they are less tolerated by children.

* The present study was based on a review of the tertiary center charts between 2010-2020. We analysed the data according to two groups: children who received a mastoid pressure dressing for 24-hours postoperatively and children who did not receive any pressure dressing.

* Evaluation of postoperative complications following mastoidectomy showed no significant differences between the groups.

* The use of MPD evidences a deleterious effect on postoperative pain, as patients with MPD reported worse VAS results.

\section{Introduction}

Mastoidectomy is one of the most common surgical procedures performed on the temporal bone ${ }^{1}$. Its indications include cholesteatoma, acute mastoiditis, chronic middle ear disease, chronic tympanic membrane perforations, tumours, and cochlear implantation ${ }^{2,3}$. Since the 1950s, the application of postoperative, mastoid pressure dressings (MPD) became widely accepted following the first use of temporalis fascia grafts in middle ear surgery ${ }^{4,5}$. The procedure for applying a mastoid pressure dressing consists of a circumferential head bandage overlying non-stick padded dressings, such as gauze swabs, which are placed over the pinna, thus applying pressure to the surgical area ${ }^{6,7}$. Mastoid pressure dressings are usually kept in place for 24 hours, replaced after one (1) day, and removed after three (3) days ${ }^{9,10}$. The ostensible purpose of this dressing is to prevent the potential formation of a dead space that may cause hematoma or seroma formation, which can result in pinna protrusion or wound breakdown and infection ${ }^{4,6,8}$.

Mastoid pressure dressings can cause severe discomfort, itching, headaches, and pressure ulcers. They are less tolerated by children, especially in hot weather, and are associated with anxiety upon removal ${ }^{10}$. In addition, the sight of a child's head wrapped in a large mastoid pressure dressing may cause parents to experience increased stress and concern.

Some prior studies that investigated the efficacy of applying mastoid pressure dressings following cochlear implantation and middle ear surgery in adults showed no significant difference in the rate of wound complications ${ }^{7,14}$. In fact, other reports revealed an increase in wound complications among patients upon whom a mastoid pressure dressing was applied after middle ear surgery ${ }^{8,13,15}$. Regardless, the efficacy issue remains unresolved, with most institutions continuing to apply mastoid pressure dressings on patients undergoing mastoidectomy, including children. In fact, no data has been published about pressure dressings applied to children following mastoidectomy in a contaminated surgical site such as that found in cholesteatoma and chronic ear disease, and their relation to postoperative pain. This is likely because a presumption has built up around this practice that it is intrinsically beneficial.

\section{Objectives}


In this study, we assess the need for mastoid pressure dressings on children to prevent wound-related complications and minimize postoperative pain following mastoidectomy surgery. To do this, we examined the visual analogue scale (VAS), which was developed to evaluate patients experiencing chronic and acute pain, particularly acute postoperative pain ${ }^{11}$.

Materials and Methods

This study was based on a review of the tertiary center charts of childhood-aged patients who, between 2010 and 2020, underwent mastoidectomy surgery. All cases were performed by senior, experienced surgeons at tertiary healthcare campus (A.G. or M.C.V.). From these medical records, we collected data regarding demographics (age and gender), diagnose, physical examinations, treatments, complications, and VAS scores for acute pain obtained during the postoperative stays.

We analysed the data according to two (2) groups: children who received a mastoid pressure dressing for 24hours postoperatively and children who did not receive any pressure dressing (where their wounds were only covered). Eligible patients for the study were children under age 18 who underwent mastoidectomy surgery for inflammatory etiology at our institution between 2010 and 2019. Exclusion criteria included: (1) bleeding disorders; (2) mastoid pressure dressing removed less than 24-hours postoperatively; (3) endaural approach mastoidectomy (4) mastoidectomy with non-inflammatory indications, such as cochlear implantation; and (5) lack of follow-up.

Complications were classified as major or minor, as described in previous studies ${ }^{16,17}$. Minor complications were defined as adverse events that were managed by medical measures, conservative treatment, or minor surgical procedures. Examples are surgical site infection (SSI), dehiscence, and seroma aspiration. Major complications included adverse events that required major surgical procedures; intracranial complications, such as meningitis; or a permanent disability, such as persistent facial paralysis.

All operations were performed with the patient under hypotensive general anesthesia and all ears were locally infiltrated, post aurally and inside the meatus with 1:200,000 adrenaline. Standardized operative techniques were used for all cases. Hemostasis was achieved using bipolar diathermy at a setting of $12 \mathrm{~W}$. Wound closure in the non-mastoid pressure dressing (NMPD) group was achieved with interrupted subcutaneous sutures, interrupted or continuous skin sutures, and $1.5 \mathrm{~cm}$ Steri-Strip ${ }^{\circledR}$ applied along the line of the incision, covered by Steripad (Figure 1 ), while in the mastoid pressure dressing (MPD) group subcutaneous sutures were followed with tissue adhesives for skin closure and Steri-Strip ${ }^{\circledR}$ application.

The MPD is composed of a circumferential head bandage with several gauze pads $(6 \times 6 \mathrm{~cm})$ and cotton wool over the pinna and the mastoid. A ribbon gauze is tied over the eyebrow to keep the bandage in place (Figure 2 ). The surgical incision was evaluated at discharge and at the first postoperative appointment, two to four weeks after surgery. For all patients, routine prophylactic, postoperative antibiotic treatment was administered systemically for seven days and locally, via eardrops, for two weeks.

\section{Ethical Considerations}

This study was conducted after its protocol was approved by our institution's Helsinki Committee review board (0742-20-RMB).

\section{Statistical Analysis}

Results are presented as mean \pm SD for quantitative variables and number for categorical variables. Fisher's exact test and t test were used for comparison of categorical and quantitative variables, respectively. All statistical assessments were two-sided and evaluated at the 0.05 level of significant difference, using IBM SPSS Statistics version 22.0 for Mac (IBM Corporation, Armonk, New York, USA).

\section{Results}

One hundred nineteen (119) patients were enrolled in this study. Age at surgery ranged from 0.5 to 17 years, with a mean age of $10.6 \pm 4.1$ years ( $73.9 \%$ male and $26.1 \%$ female). Mean follow-up time was $28.3+$ 
42.6 days. Patients were divided into two (2) groups according to the use of MPD following mastoidectomy surgery - ninety-one (91) patients in the MPD group and twenty-eight (28) patients in the NMPD group. Among the former, mastoidectomy due to cholesteatoma was performed on 83 of 91 patients (91.2\%); among the latter, mastoidectomy due to cholesteatoma was performed on 24 of 28 patients $(85.7 \%), \mathrm{p}=0.8$.

All 119 surgeries were successful. As shown in Table $\mathbf{1}$, the mean length of stay was $2.1 \pm 2.8$ days, with no significant difference between the groups, while demographic and medical characteristics were also quite similar. There were no postoperative mortalities and no documented cases of cerebrospinal fluid (CSF) leak, brain abscess, or deafness.

Regarding minor complications, there were no significant differences between the groups $(\mathrm{P}=0.47)$. In the MPD group, five (5) patients had a minor wound dehiscence, of which three (3) experienced SSI, while five (5) other patients developed SSI as a single complication. One (1) patient presented a keloid scar in the long follow up. In the NMPD group, one (1) patient had a SSI and another developed retroarticular swelling several hours after the surgery due to a local hematoma, which required a return to the operating room to identify the source of the bleeding and achieve hemostasis. Two to four weeks following surgery, no seromas, erythema, scalp folliculitis, bruising, ulcers, or skin necrosis were found in either group. With respect to major complications, neither group presented meningitis or facial paralysis (Table 2 ). As regards VAS values recorded the night of surgery and several hours before the head dressing was removed, patients in the NMPD group reported slightly better scores with a significant difference between the groups $(0.8 \pm 1.6$ and $1.9 \pm 2.4$, respectively), $\mathrm{p}=0.02$ (Figure 3 ) .

\section{Discussion}

In the early 1970s, MPDs became standard practice following their introduction by Heerman et al. for use after temporalis fascia grafts for tympanoplasty ${ }^{5}$. MPDs were thought to be useful in stabilizing and protecting wounds, preventing contamination, providing an optimal healing environment, and absorbing secretions ${ }^{18-20}$. However, several years later, early exposure of surgical wounds open air had gained popularity in many surgical fields and many surgeons had begun to wonder whether pressure dressings were necessary at all. Along these lines, the discrepancy on the use of MPDs between otolaryngologists is not surprising in light of the absence of reliable evidence that MPDs in fact reduce rates of wound complications among adults, much less that they provide benefits which outweigh complications within the pediatric population. Our study showed no statistically significant difference between the groups either in minor or major wound complications.

This retrospective investigation was conducted to determine the efficacy of MPD application following mastoidectomies performed on children and its effect on the discomfort and pain they experience, as measured by the VAS. Our study is unique because no prior study has evaluated the need (or lack thereof) for MPDs among pediatric patients undergoing mastoidectomy for inflammatory conditions, including postoperative pain scores. For this research, postoperative, wound examination data and VAS values were compared between a group of patients who underwent mastoidectomy with MPD and a group of patients who underwent the same surgeries with no MPD.

In addition, our comparison of VAS values evidenced a significant difference between the groups, with lower levels of pain in the NMPD group. It is important to mention that the VAS values were all obtained by a single assessment (during the postoperative night, several hours before MPDs were to be removed).

Due to the retrospective nature of the study the discomfort or pain during the removal of the dressing was not evaluated. More assessments of the VAS (before, during, and after MPD removal) would have given us a better appreciation of the pain caused by its removal. It is also important to note that the VAS does not consider patients experiencing discomfort without pain, nor does it measure levels of parental stress and concern while their child's head is covered with an MPD. Therefore, further prospective studies are needed to evaluate a more complete spectrum of pain and in a more accurate manner.

Albeit an insignificant finding, a trend toward longer lengths of stay was found in the NMPD group, a fact 
that may be explained by the higher percentage of mastoidectomies performed due to mastoiditis (which are typically associated with longer-term hospitalizations). This trend enhances the notion of abandoning MPD, as even with higher percentage of infectious disease as mastoiditis, fewer SSIs were seen in the NMPD group.

For the present moment, however, our findings corroborate in children the findings of previous publications on adults, that showed absence of advantages in $\mathrm{MPD}^{8,13,15}$. A prospective, randomized study conducted by Castelli et al. among 420 postauricular tympanoplasties (or tympanomastoidectomies), compared woundrelated complications between patients with MPDs and those without ${ }^{8}$. There were three (3) cases of pinna bruising in the MPD group and no cases in the NMPD group. There were no hematomas or wound infections in either group, which may be attributed to the use of modern bipolar diathermy. However, unlike the current study, there was no specific reference to pediatric patients.

Previous publications in pediatric population, focused on evaluating MPD in non-inflammatory diseases. Lou et al. performed a retrospective review in pediatric cochlear implant patients and found no statistically significant difference in hematoma formation or other wound complications between the studied groups ${ }^{7}$. A prospective trial by Powell et al. to evaluate the role of head dressings in the postoperative management of the prominent ear in a plastic surgery unit also found them to be unnecessary ${ }^{21}$. Their findings are compatible with the results of this study, although as opposed to the present investigation, they gave no consideration to pain associated with MPD, as VAS values were not reported.

Further studies, such as Rewe-Jones et al., have shown not only no obvious advantages in the use of MPDs, but several disadvantages, including an increase in wound infections ${ }^{13}$. Moreover, tight dressings may cause pressure necrosis of the skin on the lateral surface of the face or pinna ${ }^{21}$.

Some of the limitations of our study was the disparity of the sample size between groups, which is explained by the fact that patients in the NMPD group were operated only in later years. Additionally, it should be taken into consideration that there may be some element of selection bias in this work due to its nonrandomized, retrospective nature, particularly as each group of patients underwent surgery by a different surgeon. Nonetheless, with respect to the use of MPD, dressings were placed as per the proclivities of each surgeon and were not dependent on any patient's condition, nor the nature and course of their surgery, so, it is possible to assume that there was equal representation of patients in both groups.

\section{Conclusions}

Our results indicate that MPD application following paediatric mastoidectomy does not reduce wound complications and may well increase postoperative pain. Therefore, we recommend abandoning this routine practice.

\section{References}

1. Agus M, Giachetti A, Gobbetti E, Zanetti G, Zorcolo A, John NW, et al. Mastoidectomy simulation with combined visual and haptic feedback. Stud Health Technol Inform. 2002;85:17-23.

2. The open cavity mastoid operations. In: Glasscock, M, Shambaugh, G, eds. Surgery of the ear. 4th ed. Philadephia: W.B. Saunders Co., 1990: 229-47.

3. Gruening E. On the indications of opening the mastoid process in cases of otitis media purulenta acuta with implication of the mastoid cells. Arch Otol 1879; 3: 84-88.

4. Khan I, Mohamad S, Ansari S, Iyer A. Are head bandages really required after middle-ear surgery? A systematic review. J Laryngol Otol. 2015;129(8):740-3.

5. Heermann J Jr, Heermann H, Kopstein E. Fascia and cartilage palisade tympanoplasty. Nine years' experience. Arch Otolaryngol. 1970;91(3):228-4.

6. Okur E, Kahveci OK ema., Miman MC e., Yıldız H, Ayçiçek A, Altuntaş A. Ear protrusion after tympanoplasty and the use of mastoid dressing. Kulak Burun Bogaz Ihtis Derg. 2014;24(2):74-7. 
7. Luo SD, Su CY, Wu CM, Hwang CF. Mastoid pressure dressing for cochlear implantation-Is it necessary? Int J Pediatr Otorhinolaryngol. 2009;73(6):857-60.

8. Castelli ML, Tavormina P, Lisi D Di, Cappellaro E, Marcato P, Sartoris A. Is pressure dressing necessary after ear surgery. Ann Otol Rhinol Laryngol. 2001;110(3):254-6.

9. Chattopadhyay S, Biswas D, Paul SS, Jyoti J, Mishra A, Singh A. Conventional Mastoid Bandage Following Otological Surgeries - Is It Necessary? J Evid Based Med Healthc. 2016;3(24):1056-8.

10. Van Twillert B, Bremer M, Faber AW. Computer-generated virtual reality to control pain and anxiety in pediatric and adult burn patients during wound dressing changes. J Burn Care Res. 2007;28(5):694-702.

11. Myles PS, Urquhart N. The linearity of the visual analogue scale in patients with severe acute pain. Anaesth Intensive Care. 2005;33(1):54-8.

12. Boonstra AM, Schiphorst HR, Reneman MF, Posthumus JB, Stewart RE. Reliability and validity of the visual analogue scale for disability in patients with chronic musculoskeletal pain. Int J Rehabil Res. 2008;31(2):165-9.

13. Rowe-Jones JM, Leighton SE. The value of head dressings for middle ear surgery. J Laryngol Otol 1993;107(1):17-19.

14. O'Brien JX, Ruskin O, Price T. Pressure dressings in mastoid and middle ear surgery: are they necessary? A retrospective review of patient outcomes. ANZ J Surg. 2018;88(10):1047-50.

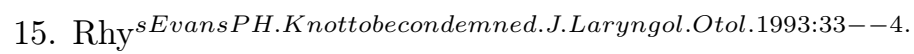

16. Stratigouleas ED, Perry BP, King SM, Syms CA. Complication rate of minimally invasive cochlear implantation. Otolaryngol - Head Neck Surg. 2006;135(3):383-6.

17. Bhatia K, Gibbin KP, Nikolopoulos TP, O'Donoghue GM. Surgical complications and their management in a series of 300 consecutive pediatric cochlear implantations. Otol Neurotol. 2004;25(5):730-9

18. Harkins, HN. Wound healing. In: Moyer, CA, Rhoads, JE, Allen, JG, Harkins, HN. Surgery: Principles and practice. 3rd ed. Philadelphia, Pa: JB Lippincott, 1965: 25-7.

19. MacLaren IF. Simplified wound care. J R Coli Surg Edinb 1963;9:61-5.

20. Norton, LW. Trauma. In: Hill, GJ. Outpatient surgery. Philadelphia, Pa: WB Saunders, 1973: 77-83.

21. Powell BWEM. The value of head dressings in the postoperative management of the prominent ear. $\mathrm{Br}$ J Plast Surg. 1989;42(6):692-4.

Table 1. Demographic and Medical Characteristics of the 119 Patients

Table 2. Postoperative Complications Following Mastoidectomy

Figure 1. Non-mastoid pressure dressing.

Figure 2. Mastoid pressure dressing.

Figure 3. Violin plot comparing overall VAS scores between the two surgical groups, obtained during the evening of the day of surgery, several hours before the dressing was removed.

\section{Hosted file}

Table 1.docx available at https://authorea.com/users/439317/articles/540300-is-thereutility-in-using-mastoid-pressure-dressing-in-children-undergoing-a-mastoidectomy

\section{Hosted file}

Table 2.docx available at https://authorea.com/users/439317/articles/540300-is-thereutility-in-using-mastoid-pressure-dressing-in-children-undergoing-a-mastoidectomy 


\section{Hosted file}

Figure 1.docx available at https://authorea.com/users/439317/articles/540300-is-thereutility-in-using-mastoid-pressure-dressing-in-children-undergoing-a-mastoidectomy

Hosted file

Figure 2.docx available at https://authorea.com/users/439317/articles/540300-is-thereutility-in-using-mastoid-pressure-dressing-in-children-undergoing-a-mastoidectomy

\section{Hosted file}

Figure 3.docx available at https://authorea.com/users/439317/articles/540300-is-thereutility-in-using-mastoid-pressure-dressing-in-children-undergoing-a-mastoidectomy 